\title{
Interprofessional relationships and communication in primary palliative care: impact of the Gold Standards Framework
}

\author{
Kashifa Mahmood-Yousuf, Daniel Munday, Nigel King and Jeremy Dale
}

\section{ABSTRACT}

Background

High quality end-of-life care in the community is achieved with effective multidisciplinary teamwork, interprofessional communication between GPs and district nurses, and early referral of patients to district nurses. These aspects of palliative care are highlighted in the Gold Standards Framework, a programme recently established in UK primary care.

Aim

To investigate the extent to which the framework influences interprofessional relationships and communication, and to compare GPs' and nurses' experiences.

Design of study

Qualitative interview case study.

Setting

Fifteen participating practices from three primary care trusts in England.

Method

Thirty-eight semi-structured interviews were undertaken with GPs, district nurses, Macmillan nurses, and framework facilitators.

Results

Adoption of the framework often resulted in earlier referral of palliative care patients to district nurses. Multidisciplinary team meetings enabled

communication for sharing knowledge, discussing management problems, and keeping colleagues informed; however, arranging and maintaining such meetings was often problematic. Nurses particularly valued formal meetings while GPs generally preferred informal ad hoc dialogue. GPs largely maintained control of the mode of multidisciplinary working. The best functioning teams used a mixture of formal and informal meetings with a relatively non-hierarchical working style.

Conclusion

Implementing the framework enabled processes of communication associated with high quality palliative care in general practice, but there was marked variation in how this worked in individual teams. In general, hierarchical doctor-nurse relationships persisted.

\section{Keywords}

communication; interdisciplinary health care team; interprofessional relationships; primary care; palliative care.

\section{INTRODUCTION}

Multidisciplinary teamwork is a defining characteristic of high quality community-based palliative care. ${ }^{1}$ Collaboration and the division of labour help to ensure that medical, nursing, therapy, social care, and other resources are appropriately enlisted to meet the needs of terminally ill patients. ${ }^{2}$ A review of continuity of care and decision making identified factors that influence the effectiveness and quality of multidisciplinary teamwork; these include training, experience and expertise of team members, the team's philosophy of care, and organisational structures. ${ }^{3}$ In particular, the quality of relationships between district nurses and GPs can influence the timeliness of referral of patients to the district nursing services, effectiveness of symptom control, and the need for acute hospital admission. ${ }^{4}$ Early referral to district nurses may facilitate establishing supportive relationships with the patient and their carers: an important factor in the provision of palliative care ${ }^{5}$ which enables psychosocial and physical needs to be addressed more effectively. ${ }^{6}$

The Gold Standards Framework is a programme aimed at facilitating primary palliative care. It is

K Mahmood-Yousuf, BA, MSc, MPhil, PhD, research fellow; D Munday, PhD, DRCOG, DipPallMed, FFARCSI, MRCGP, honorary associate clinical professor; J Dale, PhD, DRCOG, FRCGP, professor, Health Services Research Institute, Warwick Medical School, University of Warwick, Coventry. N King, BA, PhD, professor, University of Huddersfield, Department of Behavioural Sciences, Queensgate, Huddersfield.

Address for correspondence

Dr Daniel Munday, Health Services Research Institute, Warwick Medical School, University of Warwick, Coventry, CV4 7AL. E-mail: d.munday@warwick.ac.uk

Submitted: 19 November 2007; Editor's response: 29 January 2008; final acceptance: 9 March 2008. (c)British Journal of General Practice 2008; 58: 256-263. DOI: 10.3399/bjgp08X279760 
intended to help primary healthcare teams with identifying patients who have palliative care needs and to ensure that they receive high quality anticipatory care and support (Box 1). Over the last 5 years the framework has been implemented in over 3000 practices across the UK. ${ }^{7}$ Various strategies and procedures, including registers of terminally ill patients and team meetings to discuss patients' needs, are encouraged to promote communication, coordination, and continuity of care. ${ }^{8}$ However, little is known about how the programme affects communication between team members.

The current researchers recently identified considerable variation between practices in their implementation of the framework. Differences were observed in the extent of palliative care-related processes and the effectiveness of interprofessional communication. $^{9}$ High performing practices displayed a clear shared purpose among staff for palliative care, whereas minimal performing practices demonstrated little utilisation of basic processes recommended in the framework and deficiencies in interprofessional communication. Effective primary palliative care appears to require good team relationships and robust processes.

This article presents further findings from the earlier study and examines how the relationship between GPs and district nurses affects the quality of communication about patients' palliative care needs. This study also examines whether implantation of the framework led to to changes in the doctor-nurse relationship.

\section{METHOD}

An interview-based study was undertaken with members of primary care teams that had implemented the Gold Standards Framework within the previous 2 years. Full details of the methodology have been described previously. ${ }^{9}$ With the assistance of local framework facilitators, practices that had participated within three primary care trusts (PCTs) were purposively sampled to provide a varied mix of practices in terms of location, size, training status, and level of uptake of the framework as suggested by audit of their palliative care processes. Selected practices were contacted by letter and a follow up telephone call: 15 of 17 practices agreed to participate.

At each practice the GP who was identified as being most involved in coordinating palliative care was interviewed, as was the district nurse who worked most closely with the practice. In addition, other professionals involved with the framework were interviewed, and observation of practice palliative care meetings and local Gold Standards Framework meetings were undertaken where possible. In total,

\section{How this fits in}

Multidisciplinary working in primary care is essential for delivering high quality

end-of-life care in the community. In the UK this is recognised through 'quality

payments' to general practices. The quality of multidisciplinary working remains

variable in practices that have undertaken a programme to encourage a high

standard of end-of-life care. Effective primary care teams establish regular

formal meetings to discuss patients and have a mechanism for informal

meetings to discuss urgent issues. District nurses and GPs are shown to have

different priorities and styles of multidisciplinary working. Effective teams have a

relatively non-hierarchical working style rather than rigidly hierarchical structure

of decision making.

38 interviews were completed: 17 GPs, 16 district nurses, three specialist palliative care (Macmillan) nurses, and two framework facilitators (Table 1).

Data were gathered from semi-structured interviews conducted to explore interviewees' experiences of implementing the framework within their practice. ${ }^{9}$ This included questions and prompts to assess and contrast experiences of the central elements of primary palliative care: communication between professionals about terminally ill patients, and teamwork in delivering care. Interviews comprised open questions exploring whether and how the palliative care approach of the practice, including communication between GPs and community nurses, changed as a result of

\section{Box 1. Suggested methods of improving palliative care delivery. ${ }^{8}$}

Elements of the framework

C1 Communication

C2 Coordination of care

C3 Control of symptoms

C4 Continuity out of hours

C5 Continued learning

C6 Carer support

C7 Care in the dying phase
Examples of recommended processes

Maintaining a supportive care register of terminally ill patients, regular multidisciplinary team meetings, patient held records

Practice coordinator to ensure smooth running of palliative care procedures. Named GP and district nurse for each patient

Holistic assessment of symptoms. Use of symptom assessment tools

Sending details for terminally ill patients to the out-of-hours provider. Leaving drugs in the home which can be anticipated as being needed (for example, diamorphine for a syringe driver)

Audit of palliative care delivery, practice/patient-centred learning including significant event analysis

Identifying carers' needs. Keeping a carers' register. Offering practical, emotional, and bereavement support

Use of protocol or pathway to ensure good practice 
Table 1. Practice demographics.

\begin{tabular}{lccc}
$\begin{array}{l}\text { Practice } \\
\text { identifier }\end{array}$ & $\begin{array}{c}\text { Practice size } \\
\text { (number of patients) }\end{array}$ & Location & Training \\
\hline $\mathrm{A}$ & $5001-10000$ & Semi rural and mixed & Yes \\
\hline $\mathrm{B}$ & $10001-15000$ & Urban & Yes \\
\hline $\mathrm{C}$ & $10001-15000$ & Rural & Yes \\
\hline $\mathrm{D}$ & $5001-10000$ & Urban & No \\
\hline $\mathrm{E}$ & $<5000$ & Semiral and mixed & No \\
\hline $\mathrm{F}$ & $10001-15000$ & Urban & Yes \\
\hline $\mathrm{G}$ & $<5000$ & Urban & No \\
\hline $\mathrm{H}$ & $<5000$ & Rural & Yes \\
\hline $\mathrm{I}$ & $<5000$ & Urban & Yes \\
\hline $\mathrm{J}$ & $10001-15000$ & Urban & Yes \\
\hline $\mathrm{K}$ & $<5000$ & Urban & No \\
\hline $\mathrm{L}$ & $<5000$ & Semi rural and mixed & No \\
\hline $\mathrm{M}$ & $5001-10000$ & Urban & Yes \\
\hline $\mathrm{N}$ & $<5000$ & Rural & Yes \\
\hline $\mathrm{O}$ & $5001-10000$ & Yes \\
\hline & & &
\end{tabular}

implementing the framework (Box 2). Follow-up questions were formulated to gain clarification and expansion. Interviewees were encouraged to recount specific situations that reflected the strengths and weaknesses of their working relationships. Interviewees' approaches to eliciting preferred place of death with patients were also explored; results from this aspect of the study will be presented in a later paper.

The duration of interviews ranged from 30 minutes to an hour. Interviews were electronically recorded, transcribed, and checked for accuracy prior to analysis. Each practice was given a unique identifier to maintain confidentiality.

\section{Analysis}

Each practice was examined as a case unit; matrix analysis ${ }^{10}$ was used to code the interviews and key themes were identified during data extraction. Matrix cells were formed by the intersection of emergent themes and individual cases (practices). The matrix

\section{Box 2. Summary of prompts in interview guide.}

Establish how the practice became interested in the Gold Standards Framework

- Elicit information on current framework activity in palliative care

- Probe as to whether palliative care approach changed as a result

- Ascertain how the register was developed (criteria used)

Investigate whether register is making a difference

Enquire as to how it is decided when patients are in last 6-12 months of life cells were then populated with relevant text from the interviews. The degree to which interview accounts at each practice concurred with each other was determined. Particular attention was given to interviewees' perceptions relating to communication style and content, impact on work routines, and patient care. ${ }^{11}$

Responses concerning the advantages and disadvantages of different modes of communication were categorised to provide an overview of experiences related to types of interactions. Participants' levels of satisfaction with formalised meetings compared with informal means of communication were inferred from the data. A coding scheme was used to identify recurrent themes and illustrative quotes were cited to convey similar and contrasting attitudes that emerged as integral to relationships. Consistency in the approach to analysis and interpretation was maintained through a process of iterative assessment conducted by two researchers.

\section{RESULTS}

\section{Effects of participating in the Gold Standards Framework}

Primary care staff reported implementing a variety of processes aimed at formalising communication and supporting anticipatory palliative care following participation in the framework. Sharing information about patients' palliative care needs and helping staff to prioritise patient contact, such as GP home visits or district nurse assessments, were generally viewed as desirable. Timely contact was seen as reassuring to patients and relatives, inspiring trust and confidence in the clinical team. As one GP (practice I) summarised: 'Dying patients should not have to struggle with access to healthcare amidst anxieties about their prognosis'.

Several district nurses reported that their involvement with patients who had palliative care needs was often contingent upon receiving information from GPs. They reported that following implementation of the framework such information reached them sooner:

'We are not automatically informed ... district nurses aren't generally informed ... but I think the GPs are much more aware now across the board that we are there to see them [patients with palliative care needs] when they are well, and they don't hang on to them until they are really poorly.' (district nurse, practice G)

This enabled nurses to establish a rapport with patients and their carers earlier, before they became too debilitated: 
'Before the GSF I wouldn't be aware of them before l'd seen them [patients with palliative care needs] ... [the GPs] would most likely only tell me if they thought that I would need to be involved, whereas now I am aware of them whether they think I need to or not.' (district nurse, practice D)

District nurses felt that patients were more likely to share social, spiritual, and emotional needs if a relationship was established earlier in the disease trajectory. Furthermore, it was hard to approach a dying person to discuss issues such as preferred place of death on a first assessment.

In one small practice a district nurse identified that being involved in the framework had led to enhanced patient care with more emphasis on teamwork:

'[Prior to adopting the framework] a lot of the care was just managed by the district nurses and there was very little involvement from the GP ...

But since the GSF was introduced we've had very good relationships where we've done joint visits with the GPs and seen patients and discussed them.' (district nurse, practice I)

The GP at the same practice agreed that the district-nurse role is a 'major link' in caring for palliative care patients. He explained how he made himself available for the district nurse:

'The district nurses if they need anything ... can pop in between patients and talk to me directly so they're not bound to ... wait until a meeting. So ... there isn't that ... barrier ... There isn't a time set and the district nurse knows that she can come and talk to me straight away or ring me up.' (GP, practice I)

Discussing individual patients together, in detail and in person, not only improved awareness, understanding and management of their condition, but also strengthened professional relationships between team members:

'It was quite a very close situation with the male doctor in the practice... He was sharing with me how he had dealt with this terminally ill girl and ... I feel that our relationship did improve because of that ... they'd bared their chest ... they shared good and bad that happened with the patient ...' (Macmillan nurse 3)

\section{Formal processes of communication:} benefits and drawbacks

There were contrasting views about the extent to which the processes implemented through adherence to the framework aided or detracted from practices' provision of palliative care. Some interviewees felt that it formalised the high standard of care already being provided (district nurse, practice $\mathrm{D}$ ), while others felt that the framework provided useful checklists which prompted necessary care:

'Tick boxes ... make sure you know everything's been done, the patient's comfortable in their last few days.' (district nurse, practice A)

However, some responded that administrative procedures could distract the team from providing direct patient care:

\begin{abstract}
'Introducing ... tranche after tranche after tranche of paperwork and pro formas ... detracts quite often from what people have always been doing. It makes it very formulaic ... box ticking. I spend most of my time on consultations tapping my piano, my keyboard, rather than being able to look at a patient and have good eye contact.' (GP, practice C)
\end{abstract}

Some GPs complained that: 'The form filling was a complete disincentive' (GP, practice D) and served more of a bureaucratic rather than a clinical need (GP, practice N). Although the framework assumes that formal discussion, planning, and documentation are vital to enhancing the quality of communication and decision making, at least one district nurse felt her practice provided equally good care despite having no register and meetings to aid the discussion of patients:

'No one seems to fall through the net ... We are following the principles [of the framework] but not doing the documentation ... not writing in the newly diagnosed cancer patients ... We're discussing the patients.' (district nurse, practice M)

\section{Multidisciplinary team meetings}

Multidisciplinary team meetings were generally valued, and were felt to provide a formal channel of communication for sharing knowledge, discussing treatment, and keeping colleagues informed. GPs felt that nurses valued team meetings because it: 'boosted their confidence and made them feel that they were needed and that they were wanted' (GP, practice J).

One framework facilitator, a GP, reflected on the value of the framework in transforming meetings from 
administratively-focused events, which did not tend to include a clinical agenda, into opportunities for discussing patients and consolidating relationships between GPs, district nurses, and Macmillan nurses:

'It was just a huge meeting and ... it was very much ... about how do we work as a practice, rather than discussing clinical issues ... The district nurse was never involved. What we suggested was ... inviting the district nurse ... to discuss patients on the register ... They send their Macmillan nurse a timetable of practice meetings when the register is going to be discussed so that they can attend as well when possible.' (facilitator 1)

The information shared at these meetings meant that all practice staff became aware of patients receiving palliative care, encouraging a multidisciplinary approach to management. As reiterated by another facilitator (a specialist nurse):

'The central features of GSF for me is that it has to be a whole practice approach and that means you don't leave it to the receptionist there, or the practice nurse or the district nurse, you have to include the whole primary healthcare team ... There is this hierarchy ... the GP is at the top, and "I'm only a district nurse", the way you are spoken to ... has improved by them actually sitting down with the GPs and building up a relationship with them.' (facilitator 2 )

For district nurses, formal meetings compensated, at least partially, for the difficulties of accessing GPs which occurred in normal day to day practice.

'GPs ... after their surgery they're rushing off to do their visits ... You can't pin them down; whereas a meeting is ... time out isn't it? So that the atmosphere is more relaxed, without seeing a patient.' (district nurse, practice $\mathrm{H}$ )

However, several practices found it difficult to organise multidisciplinary meetings because of organisational constraints. Sometimes, district nurses did not attend meetings, even when they were invited. One GP complained that there had been no meetings since a change in district nursing occurred 4 months earlier: 'despite protests' (GP, practice J); while the district nurse confirmed this was one of the: 'Stipulations that isn't working so grand at the moment ... it's at a standstill' (district nurse, practice $\mathrm{J}$ ).

Changes in personnel, staff shortages, workload pressures, and inconvenient times of meetings were factors that contributed to difficulty in attending. In addition, as one district nurse (practice $\mathrm{M}$ ) reported that information about meetings sometimes only appeared on the day it was scheduled, which made it unlikely that she could attend.

In small practices, because few palliative care patients are likely to be cared for at any one time, regular meetings might not be feasible. In one such practice their solution was to hold meetings after a patient had died to discuss and reflect on the care provided (GP, practice G).

\section{Informal communication}

GPs tended to value informal communication with district nurses much more highly than formal meetings. District nurses were recognised as often being better informed about patients' day to day condition, and so could alert the GP when a patient is deteriorating and might require greater attention:

'The nurse would probably be a lot more switched on, sensitive to the home circumstances and the family ... The doctors would probably be more up to speed in terms of any developments as regards the state, the progression of the disease and illness.' (GP, practice M)

However, both GPs and district nurses could be satisfied with an informal mode of communication, whether the conversation took place in the corridor or the consulting room. This was felt to be: 'Much more ideal than formal meetings' (district nurse, practice $M$ ), because issues were discussed without delay at the most relevant point in time.

\section{Challenges to teamworking}

Some starkly contrasting views emerged about the quality of teamwork within the same practice. In one small practice, GPs worked in the surgery at alternate times which made it difficult to organise meetings with the district nurse. Although the nurse felt supported, she was dissatisfied by the inability to meet with the GPs as a team and share concerns and ideas:

'I felt very supported, but they wouldn't meet as a group with me ... I just felt that I couldn't discuss my fears with all of them ... if you needed more drugs, more anti-emetics, or more morphine ... you needed them to come out with you and do a visit ... I felt the support wasn't as great, though I could see any of them at any time ... we never discussed a patient after they died ... I don't feel we learnt from each other.' (district nurse, practice $\mathrm{H}$ ) 
By contrast, a GP from this practice was satisfied with these informal interactions, reporting that they did have formal team meetings:

'We always meet every day at coffee time anyway 'cause we are a very small team ... And we formally meet on a 1 to 3-monthly basis with all of the staff ... but then we see the district nurses every day anyway ... It's much more ideal than formal meetings.' (GP, practice $\mathrm{H}$ )

Specialist palliative care nurses reported particular challenges to effective team working, giving accounts of how they persevered in attempting to develop more communicative relationships with GPs and district nurses. Despite reaching out to practices, one such nurse claimed she had only been invited to one meeting:

'I've been ... ringing up the practices ... asking them ... if they need any help, when they've got a practice meeting - let me know, so I can go ... which l've ever been to one meeting ... I've had to ring up and remind them that l've wanted to come ... sort of check up on them all ... I haven't heard from them for a while about a meeting.' (Macmillan nurse 1)

However, this nurse also identified how seeking closer involvement with practices could lead to becoming over stretched:

'It's going to be hard isn't it, but I think it's going to be every 6 months then, or as necessary.' (Macmillan nurse 1)

Another specialist nurse depicted the varied impressions she had formed about the range of practices she worked with. One of them, for example, was portrayed as being highly motivated and very inclusive, as she was consistently invited to their meetings. However, other practices were slow to respond to such requests:

'I've approached the practice manager and asked to invite me to GSF meetings and said I'm keen to come and get involved and so far they haven't done that ... I need to get back to all these people and remind them that I exist.' (Macmillan nurse 2)

As well as reporting on issues arising from organisational aspects of palliative care teamwork, specialist nurses also differentiated between GPs, for instance, according to their communication, level of active cooperation, and team spirit:
'GPs tend to fall in my mind into several categories, those that would listen ... and would facilitate... Then you have GPs that almost block you, that don't maybe communicate with you ... and then there's the other ones that fall inbetween, in that they would listen to you and would maybe prescribe or not ... It depends on how much we can be a threat to them really in that some GPs are really very good on their palliative care and would be able to talk one to one, but I think it comes down to personality in lots of ways ... their ability to accept information from another role.' (Macmillan nurse, 3)

\section{DISCUSSION}

\section{Summary of main findings}

This study illustrates a range of experiences and perceptions concerning the communication between GPs, district nurses, and specialist palliative care nurses following the implementation of the Gold Standards Framework, a programme that emphasises the importance of an anticipatory multidisciplinary approach to primary palliative care.

Most interviewees agreed with the principles of the framework, and many reported that it had brought benefits to the care of terminally ill patients, particularly in terms of enhanced teamwork and better communication. While some reported that using the documentation associated with the framework had improved care, others thought this had been burdensome or had detracted from a patient-centred approach.

Although there was universal acceptance of the need for interprofessional communication, there were different perspectives about the relative merits of formal multidisciplinary meetings and informal meetings between practitioners when the need arose, with district nurses in general preferring the former and GPs the latter.

While some challenges associated with GP and district nurse communication and interprofessional working were reported, specialist nurses were particularly aware of a significant variation in both the willingness of practices to involve them in meetings and in the attitudes of GPs towards them as coworkers.

\section{Comparison with existing literature}

This study confirms and builds on the finding of King, et al ${ }^{12}$ who reported on a qualitative evaluation of a pilot phase of the framework involving 76 practices. They identified that in practices implementing the framework, communication was improved, processes strengthened, and terminally ill patients were less likely to be overlooked within the practice. Similarly they noted the burden of 
increased paperwork as being a major drawback to the framework and highlighted the importance of stable and well functioning primary care teams in enabling the principles of this initiative to be achieved. The study reported in this paper included practices selected from 1300 practices enlisted in the first national roll out of the framework and has therefore confirmed its utility beyond the pilot stage, albeit still among early adopters. In addition, this study has explored in more depth the dynamics of interprofessional relationships and has illustrated that the framework is used in a variety of ways within individual practices.

As has been reported elsewhere, ${ }^{5}$ early referrals were identified as enabling district nurses to develop a relationship with patients earlier in the illness trajectory, increasing the likelihood of providing more holistic care that can meet psychological and spiritual as well as physical needs. Early referral also promotes greater continuity through forward planning and information sharing.

Formal meetings enabled a review of all palliative care patients, irrespective of their most recent contact. These meetings offered time for reflection, education, and teambuilding. Conversely, identifying patients' needs explicitly and communicating them between team members in a timely fashion, often during informal meetings between GPs and district nurses, enabled patients to be prioritised for GP home visits or nursing assessments. This important informal communication could be put at risk, as practicebased district nursing services in the NHS may be increasingly threatened by the 'corporatisation' of district nurses into area teams.'

Kraut et $a /^{13}$ argued that informal communication reinforces organisational and group coordination, especially under conditions of uncertainty. It is useful in supporting the social functions of groups and maintaining their good will, transmitting organisational culture, and helping to solve problems efficiently. Where formal and informal meetings were in operation, communication appeared to be working most effectively. However, changes in personnel could lead to a disruption in the functioning of meetings. This might indicate that different healthcare professionals place a higher or lower value on meetings, or it may highlight the importance of time and informal relationships needed for the development of networks or 'communities of practice' 14 within primary care teams.

The operation of power in the relationships of GPs and district nurses and how this affected patient care was recently reported by Speed and Luker. ${ }^{15}$ Their study highlighted evidence of similar dynamics between GPs and district nurses and also GPs and specialist nurses in caring for palliative care patients. While there were some examples where the hierarchical nature of the primary healthcare team had been flattened, enabling more equal multidisciplinary team work approaches to emerge with district nurses and GPs undertaking joint visits and learning together as they reflected on experiences, these were uncommon.

The imbalance of power was most clearly illustrated by several district nurses describing situations where they had difficulty in accessing GPs for advice or support. Similarly, specialist nurses reported that it could be difficult to achieve the cooperation of some GPs; however, there was no example of GPs expressing concern about the need to wait on the district nurse's convenience to have a conversation. Similar persistent echoes of the nurses' traditional role as the 'handmaiden' of the doctor have been noted elsewhere. ${ }^{15-17}$

Long explored the experience of teamwork in primary care and concluded that the clinical and legal responsibility and status of doctors as employers (or commissioners of services in the case of district nursing) can affect team members' perceptions concerning a steep hierarchical structure with sharp differences in status and rewards. ${ }^{18}$ While hierarchy or power distance can inhibit communication, effective leaders (GPs) can flatten out these structures making communication from less powerful members (district nurses) safe and effective. ${ }^{19}$ Professional identities, power, and organisational structures are inevitably intertwined, and each needs to be acknowledged and addressed to ensure effective teamwork in primary care. In addition, subtle interprofessional perceptions have also been shown to affect referral between team members. ${ }^{20}$

Finally, high quality teamwork does not only affect patient care directly but may also be beneficial for the mental health of its members which, in itself, is important for the maintenance of well-functioning teams. ${ }^{21}$ Conversely, individual personality clashes or group conflict can occur between GPs and community nurses. This can create divisions and dysfunction in the team which can be reflected in delivery of substandard care. ${ }^{15}$

\section{Strengths and limitations of the study}

This study explores the issue of interprofessional communication in community palliative care and the impact of the Gold Standards Framework: areas in which research to date has been sparse. Using a case-study approach enabled perceptions of GPs and district nurses working in the same practice to be compared. This facilitated an exploration of 
dynamic relationships within the practices. However, in most practices only the lead GP for palliative care and one district nurse were interviewed, and their experiences and opinions may not have been representative of the whole practice. In addition, it is unclear how closely their reports reflected the actual situation within the practice.

All practices in this study were early adopters of the framework and had taken up the programme before any financial incentive was provided to maintain registers of palliative care patients, or to conduct multidisciplinary meetings at which these patients were discussed. They may, therefore, not be representative of practices in general, particularly later adopters.

\section{Implications for future research and clinical practice}

Since this study was completed, the Gold Standards Framework has been formally adopted by more than $30 \%$ of general practices in the UK. Many other practices are also following the process of maintaining registers of palliative care patients and conducting regular multidisciplinary meetings to discuss them, as recommended in the Quality and Outcomes Framework of the general medical services contract. The findings of the current study suggest that, while the Gold Standards Framework can assist practices in the delivery of primary palliative care, achieving the highest standards in primary care teams is likely to be limited unless attention is paid to interprofessional relationships as well as processes.

Further research to explore the effectiveness of the framework in later adopters is required. This should include studies to explore in depth the practice context, professional relationships, and levers and barrier to the delivery of successful palliative care. Suitable methods could include participant-observation studies within practices and further comparative case studies of participating teams. Action research would also be a useful method, allowing primary care teams to explore their own practice and the effects of interventions undertaken.

\section{Funding body}

This study was funded as part of the Macmillan Gold Standards Framework evaluation project by Macmillan Cancer Support

\section{Ethical approval}

MREC approval for this study was obtained (05/Q2803/48) and appropriate primary care trust research governance permissions were obtained

\section{Competing interests}

The authors have stated that there are none

\section{Acknowledgements}

We would like to thank all those who participated in the research, Ms Shona Agarwal for undertaking several of the interviews, Dr Janice Koistinen for administrative support, and members of the Macmillan Primary Palliative Care Research and Evaluation Group, Chair Professor Jane Maher, for helpful comments during the conduct of this study.

\section{Discuss this article}

Contribute and read comments about this article on the Discussion Forum: http://www.rcgp.org.uk/bjgp-discuss

\section{REFERENCES}

1. Forrest S, Barclay S. Palliative care: a task for everyone. Br J Gen Pract 2007; 57(539): 503.

2. Peppiatt R. Palliative terminal care. Br J Gen Pract 1998; 48(431): 1297-1298.

3. Bliss $\mathrm{J}$, While A. Decision making in palliative and continuing care in the community: an analysis of the published literature with reference to the context of UK care provision. Int J Nurs Stud 2003; 40(8): $881-888$.

4. Austin L, Luker K, Caress A, Hallett C. Palliative care: community nurses' perceptions of quality. Qual Health Care 2000; 9(3): $151-158$.

5. Luker K, Austin L, Caress A, Hallet CE. The importance of 'knowing the patient': community nurses' constructions of quality in providing palliative care. J Adv Nurs 2000; 31(4): 775-782.

6. McIlfatrick S. Assessing palliative care needs: views of patients, informal carers and health care professionals. J Adv Nurs 2007; 57(1): 77-86.

7. Munday D, Dale J. Palliative care in the community. BMJ 2007; 334 (7598): 809-810.

8. Thomas K. The Gold Standards Framework in community palliative care. Eur J Palliat Care 2003; 10(3): 113-115.

9. Munday D, Mahmood K, Dale J, King N. Facilitating good process in primary palliative care: does the Gold Standards Framework enable quality performance? Fam Pract 2007; 24(5): 486-494.

10. Nadin S, Cassell C. Using data matrices. In: Cassell C, Symon G (eds). Essential guide to qualitative methods in organizational research. London: Sage Publications, 2004: 271-287.

11. King N. Using templates in the thematic analysis of text. In: Cassell $\mathrm{C}$, Symon G (eds). Essential guide to qualitative methods in organizational research. London: Sage Publications, 2004: 256-270.

12. King N, Thomas K, Martin N, Bell D, Farrell S. 'Now nobody falls through the net': practitioners' perspectives on the Gold Standards Framework for community palliative care. Palliat Med 2005; 19(8): 619-627.

13. Kraut RE, Fish RS, Root RW, Chalfont BL. Informal communication in organisations: form, function and technology. In: Oskamp IS, Spacapan S (eds). People's Reactions to Technology: In Factories, Offices, and Aerospace (Claremont Symposium on Applied Social Psychology). Sage Publications, 1990: 145-199.

14. Honeyman A. Communities of Practice. Br J Gen Pract 2002; 52(481): 621-622.

15. Speed S, Luker KA. Getting a visit: how district nurses and general practitioners 'organise' each other in primary care. Sociol Health Illn 2006; 28(7): 883-902.

16. Benger JR, Hoskins R. Nurse led care: nurses are autonomous professionals delivering expert care. BMJ 2005; 330(7499): 1084.

17. Nolan M. Has nursing lost its way? Towards an ethos of interdisciplinary practice. BMJ 1995; 311(7000): 305-307.

18. Long S. Primary health care team workshop: team members' perspectives. J Adv Nurs 1996; 23(5): 935-941.

19. Leonard M, Graham S, Bonacum D. The human factor: the critical importance of effective teamwork and communication in providing safe care. Qual Saf Health Care 2004; 13(Suppl1): i85-90.

20. Walshe C, Todd C, Caress A-L, Chew-Graham C. Judgements about fellow professionals and the management of patients receiving palliative care in primary care: a qualitative study. $\mathrm{Br} \mathrm{J}$ Gen Pract 2008; 58(549): 264-272.

21. Firth-Cozens J. Celebrating teamwork. Qual Health Care 1998; 7(Suppl): s3-7. 